\title{
The Language is the Tree, the Dialects are Its Lymph
}

\author{
Diana Kastrati \\ University of Tirana, Faculty of foreign languages, Departement of Italian, \\ dianakastrati@yahoo.it
}

\author{
Aida Gjinali \\ University of Tirana, Faculty of foreign languages, Departement of Italian, \\ aidagjinali@yahoo.it
}

Doi:10.5901/jesr.2014.v4n4p225

\begin{abstract}
Sociolinguist reflections on the balance language/dialect relying as a point of reference on the book: "The tongue goes where the tooth aches" Tullio de Mauro, the Italian linguist well known in Europe, in an unusual but interesting book published in Italy in November 2013, discusses on the famed issue language/dialect with the other contemporary Sicilian writer, also internationally known, Andrea Camilleri. The free standings of both interlocutors, each one in its position, respectively as an academic linguist and writer, brings a sum of very important conclusions regarding the argument mentioned above. In the light and optics of some of these conclusions, we will try to look more closely at how the current situation in Albania appears on this report. The heated debate in academic and non-academic districts on the possibility of reviewing the standard Albanian language, has brought an unprecedented cacophony of opinions. This debate has brought the need to really understand what the language is and what the dialect is. What is our approach to one or another? Till what point is it permissible to include in this debate the media or the massive non-specialized population in this field? Is there a risk that the "politicized" or even simply passionate treatment of this issue will have consequences on the future of the Albanian standard language or its dialects? Thus, we will try to see all this report through the clear sociolinguistic optics. This will constitute the focus of this study venture.
\end{abstract}

Keywords: standard language, dialect, linguistic variety, linguistic comunity, regional languages, academic debat, mediatic debat.

\section{De Mauro e Camilleri sul Rapporto Lingua Standard/Dialetti}

La questione del rapporto indissolubile lingua standard/dialetti è presente in tutte le realtà linguistiche a livello internazionale. Senza nessuna esitazione la realtà sociolinguistica italiana costituisce un caso ricchissimo di spunti di riflessioni in merito. Nell'ambito di queste riflessioni ed opinioni risulta estremamente interessante la pubblicazione in Italia nel novembre del 2013 di un libro intitolato "La lingua batte dove il dente duole" (Editori laterza, 2013) a cui faremo riferimento in questa sede. L'opera, scritta a quattro mani da Tullio De Mauro, noto linguista contemporaneo di renomé europea e Andrea Camilleri, altrettanto noto e prolifico scrittore italiano contemporaneo, risulta una esposizione di taglio non tanto accademico quanto divulgativa esplicativa. La loro discussione disinvolta da operatori della lingua in questo caso, relativamente dello studioso e dello scrittore, tocca i punti più piccanti dell'uso e ruolo della lingua standard e dei dialetti in Italia. In una esposizione fresca e ricca di contenuti, vorremmo soffermarsi su alcuni aspetti rilevanti dell'opera in questione le quali costituiranno anche le principali tesi del nostro intervento:

"Sono convinto che lo stato di salute di una lingua corrisponda allo stato di salute di una nazione [...]".

"Nell'epidermide di un uomo si possono trovare, sopra, le ferite superficiali, vergate in italiano, in francese, in latino; sotto ci sono le ferite più antiche, quelle delle parole del dialetto che rimarginandosi hanno fatto delle crost. Queste ferite se toccate, provocano una reazione a catena, difficile da spiegare a chi non ha il dialetto. C'è un nocciolo indistruttibile di materia, presa coi tralci prensili dei sensi; la parola del dialetto è sempre incavicchiata alla realtà, per la ragione che è la cosa stessa, percepita prima che imparassimo a ragionare, e immodificabile anche se in seguito ci hanno insegnato a ragionare in un'altra lingua".

"Il dialetto è sempre la lingua degli affetti, un fatto confidenziale, intimo, familiare. Come diceva Pirandello, la parola del dialetto è la cosa stessa, perché il dialetto di una cosa esprime il sentimento, mentre la lingua di quella cosa esprime il concetto. [...] A me con il dialetto, con la lingua del cuore che non è soltanto del cuore ma qualcosa di ancora più complesso...è una questione di cuore e di testa."

"Quando il discorso si fa serio, si usa il dialetto. Lo slittamento verso il dialetto in quel caso non è emotivo."

"La lingua si muove come una corrente: normalmente il suo flusso sordo non si avverte, perché ci siamo dentro, 
ma quando torna qualche emigrato si può misurare la distanza dal punto dove è uscito a riva."

"Tutto può cambiare, ma non la lingua che ci portiamo dentro, anzi che ci contiene dentro di sé come un mondo più esclusivo e definitivo del ventre materno. (Italo Calvino)"

"In me rimane la speranza e l'augurio che i dialetti non spariscano del tutto, che possano in qualche modo sopravvivere. Magari come qualcosa DOP, di origine protetta." (De Mauro, Camilleri, 2013.)

"Dal mio punto di vista la lingua è tutto. Ė il modo di comunicare che hanno gli appartenenti a una nazione, è il terreno comune che adoperiamo per comprendere ciò di cui stiamo parlando. [...] Pensa alla spedizione di Garibaldi...a tutte quelle persone provenienti da tante regioni diverse che non si capiscono tra loro e che in due tre giorni di navigazione diventano un esercito... È il miracolo compiuto dal comune ideale, dal comune obiettivo, dall'intesa che c'è fra queste persone. Così vedo la lingua italiana: ciò che ci fa raggiungere degli scopi comuni... Se l'albero è la lingua, i dialetti sono stati nel tempo la linfa di questo albero."

\section{II Ruolo Sociolinguistico ben Definito dello Standard e dei Dialetti}

Ci corre l'obbligo di chiarire ulteriormente che il nocciolo del problema sta nel fatto che ogni idioma 0 dialetto è potenzialmente una lingua quanto alla uguaglianza dell'organizzazione grammaticale. Sia la lingua, chiamata così per ragioni storiche e sociali, sia i dialetti hanno una grammatica rigorosa. La differenza sta nel loro uso sociale. In sociolinguistica il termine "standard" è polisemico, il quale può coincidere 0 no con una varietà socio-geografica particolare di lingua usata da un gruppo o comunità di parlanti. Per cui, questa nozione "standard" starebbe ad indicare un insieme di tratti socialmente marcati della lingua, paralata in genere da parlanti colti. Capita però che questo concetto si sovrapponga un altro: a quello della lingua nazionale (Berrutto G., 2006). L'italiano standard per esempio è naturalmente basato sulla varietà tosco-fiorentina, ma non coincide totalmente con essa: si tratta in effetti di una varietà in parte artificiale, che non è appresa praticamente da nessun parlante come lingua materna, e che vale come modello normativo (Berrutto G., 2006). Secondo gli studiosi si può definire come standard una lingua che adempie almeno queste funzioni: stabilità flessibile; intellettualizzazione, adattamento alla produzione dei testi astratti; funzione di norma codificata; funzione di prestigio per chi lo parla; funzione unificatrice in quanto serve al legame tra parlanti di varietà socio-geografiche diverse della stessa lingua rendedndoli compartecipi della stessa comunità; funzione separatrice in quanto simbolo di unità nazionale distinta dalle altre.

Partendo da queste funzioni sopra citate possiamo dedurre che la varietà dello standard è sempre parlata dall'élite socio-culturale delle classi dominanti attraverso la scuola in primis, e poi da tutte le istituzioni pubbliche e dai mass media.

Quanto al concetto sociolinguistico del dialetto, possiamo rammentare il fatto che è sempre subordinato allo standard nel repertorio linguistico di cui sono membri, cioè di una lingua. Dunque, tra lingua e dialetti non esiste nessuna differenza linguistica, solo sociale. Alcuni attributi di questa variente consistono nell'uso limitato dei parlanti, dunque nella sua oralità; la sfera dell'uso prevalentemente familiare, confidenziale e intima. Dall'altro canto il dialetto non gode gli stessi standard sopra accennati caratterizzanti invece lo standard, tra cui quello del prestigio. II dialetto appare così molto relativo il quale potrebbe incorporare anche altre caratteristiche se usato in una certa comunità. Questi valori aggiunti del dialetto in rapporto alle altre varietà che coesistono, possono variare e molto, diventando ostacolanti per l'unità linguistica di una comunità linguistica.

\section{L’Albania e il Dibattito Scientifico e "Nazional-Popolare" sullo Standard e i Dialetti}

Lo sviluppo attuale dello standard albanese e delle sue varianti va visto entro la cornice dello sviluppo generale politico e sociale della società albanese. Nel 1972 ebbe luogo il Congresso dell'Ortografia ion cui si sancì definitivamente la cornice normativa della lingua standard. Queste norme sono ancora in vigore. Accanto allo standard esistono anche due dialetti, quello gegë parlato nella zona del nord Albania fino a Shkumbin (il nome del fiume) nel centro, e da Shkumbin in giù il dialetto toskë. Lo standard si fondò basandosi maggiormenete sul dialetto toskë. Risulta evidente che la motivazione dell'attuazione di questa norma fu basata su diversi fattori quali: la lingua standard come mezzo di controllo sociale, cioè la normalizzazione di connotazioni e dei valori in modo da influire anche sul pensiero dei parlanti (Klein G.B., 2006); l'integrazione nazionale specialmente rilevante nei regimi totalitari (quale il nostro dell'epoca) ecc. Da un'ottica sociolinguistica, la pianificazione linguistica doveva puntare in primis ad individuare e classificare la variazione del comportamento nei confronti della lingua. Si presuppone che andava fatta una decomentazione dettagliata su come ci si giunge alle decisioni sull'ortografia o sulla scrittura della lingua nazionale, per confrontare poi le diverse reazioni provocate 0 perseguite dalle istituzioni all'interno e dall'esterno del governo, se fossero state perseguite, accettate 0 
respinte in modi diversi dalla popolazione. C'è da sottolineare il fatto che il potere politico, ideologico, sociale e culturale era tutt'uno nell'Albania dell'epoca del Congresso. Un regime totalitare che normalmente non teneva conto delle tensioni sociali, regionali i quali indispensabilmente si rispecchiano anche nei conflitti linguistici. La variante gegë rivendicava il diritto di essersi stata trascurata. Di conseguenza, furono accettate tacitamente le condizioni poste dal potere. Non va dimenticato che in ogni processo politico-linguistico vi sono i gruppi di interesse che esercitano delle pressioni sugli organismi decisionali stessi.

Ovviamente le norme dello standard furono applicate grazie ad un massiccio e doveroso uso in cui giocarono uno spiccato ruolo l'istruzione, i mass media e la letteratura artistica. Secondo lo studioso della sociolinguistica albanese, prof. Gjovalin Shkurtaj (Shkurtaj Gj., 2003), "solo una lingua unica, una varietà dello standard con una norma ortografica comune per tutti i connazionali albanesi [...] può affrontare i bisogni in crescendo dell'esprimersi degli albanesi...". Inoltre, in aiuto all'elaborazione ulteriore dello standard viene sempre la letteratura artistica. Perifrasando un detto di Umberto Eco, possiamo dire che la lingua va dove vuole, ma è sensibile ai suggerimenti della letteratura. A questo punto possiamo chiederci? Quanto la letteratura artistica albanese abbia influenzato l'arricchimento della lingua? Ci sono stati spiragli di respiro anche per l'altra variante dialettale gegë? Alla luce dell'opinione sopra citata che "lo stato di salute di una lingua corrisponde allo stato di salute di una nazione", possiamo considerare la nostra lingua veramente sana?

Da una decina d'anni a questa parte in Albania si è aperto un dibattito abbastanza acceso sulla revisione dello standard con l'eventuale inserimento degli elementi della variante trascurata all'epoca, gegë. II dibattito si consuma tra specialisti del campo e non, tra opinionisti di seconda mano in sterili trasmissioni televisive e quelli dei media scritte. Tra realtà dei fatti e leggende urbane, la questione ha assunto dimensioni esagerate, politicizzandola e mettendo in serio rischio il nucleo del problema. Ciò ha portato ad una visione storta della questione allontanandosi sempre di più dalla reale e necessaria soluzione laddove fosse necessario. La manipolazione a volte dei fatti, il linguaggio acceso della rivendicazione dei diritti da parte dei rappresentanti della variante gegë, l'amatorismo interpretativo hanno conquistato il terreno prendendo il sopravento. La funzione della lingua quale unificatrice di una nazione, sta diventando un pomo di discordia tra nord e sud del paese.

Rifacendosi ai giudizi espressi da De Mauro e Camilleri nell'opera sopracitata, abbiamo ravvisato i profili sia della lingua standard che dei dialetti. Inoltre, la scienza della sociolinguistica ha già detto la sua in merito. La questione del rapporto lingua/dialetti, costituisce forse uno dei pochi argomenti consolidati ed unanimi da parte di tutti gli studiosi del campo. Sotto la luce di tali norme, quanto sia legittimo che questo dibattito coinvolga anche gli elementi non competenti del campo? Non ci sono dubbi che solo le istituzioni specializzate hanno il sacro santo diritto di sigillare le norme dopo un accurato studio ad hoc. Tutto il resta sconvolge inutilmente l'opinione pubblica deformando spesso i pareri degli specialisti.

Ci sono precedenti in altri paesi della revisione dello standard? Si. La lingua è un organismo vitale e vivente e di conseguenza ha un continuo bisogno ad essere aggiornata "aggiustata' e revisionata a dovere. Le norme stabilite devono valere ed essere applicate riferite all'intera comunità linguistica e non solo ad una parte di essa. Per cui, c'è da stare attenti all'adeguamento alla norma e ai fattori che ne influiscono quali, il prestigio e il potere di chi stabilisce la norma, sanzioni o premi, il conformismo e l'opportunismo momentaneo politico. In questa ottica, "la salute" della coesione politica albanese, gioca un ruolo di primo piano. A tal punto, il rischio della politizzazione della questione è abbastanza alto. Un'interpretazione di spunto politico o passionale mette in serio rischio il futuro della lingua. Peggio ancora se a queste interpretazioni e posizioni, vengono aggiunte quelle del provincialismo o regionalismo malato.

Dall'altro canto, i dialetti resistono ancora tramite l'uso opportuno fatto dagli operatori di essi, quali scrittori, poeti e traduttori. Questo gruppo dovrebbe consolidare una larga adesione alla cultura intellettuale, artistica e scientifica, alla buona informazione, al teatro, alla musica, al cinema, ai libri, o "almeno al rispetto per il sapere critico, storico e scientifico. Ma è proprio qui che le note si fanno dolenti". (De Mauro T., Camilleri A., 2013). Le lingue nascono dai dialetti e si nutriscono da loro. Ci viene in aiuto l'esempio del latino. Nel 390 avanti Cristo a Roma che era un paesotto, arrivarono i galli i quali misero a ferro e fuoco la città cacciando via gli abitanti. Roma, la futura Urbe era questa, eppure il dialetto di quel paesotto diventò il latino. Fu grazie a quel dialetto che in seguito nacquero e si svilupparono le lingue. Però, sarebbe impensabile pensare che la lingua possa reggersi da sola senza la linfa dei dialetti. Loro riempiono di sangue questo grande tronco chiamata lingua. Concludiamo con le parole del De Mauro: "Il dialetto non è solo la lingua delle emozioni. L'ho capito in Sicilia, da non siciliano quando sono arrivato lì professore all'università, accolto dalle famiglie dei colleghi. Si partiva con l'italiano, nel senso che tutti parlavano in italiano. Ma appena la discussione si accendeva - e quando c'era Sciascia capitava spesso - e magari si passava alla politica, improvvisamente cambiavano registro linguistico. Un po' alla volta slittavano nel dialetto, e dell'italiano si scordavano. Gli uomini, per parlare di argomenti più impegnativi intellettualmente, usavano il dialetto. Perché a Venezia come a Palermo, quando il discorso si fa serio, si usa il dialetto." 


\section{References}

Berruto G., Fondamenti di sociolinguistica, Editore laterza, Roma-Bari, 1995.

Berruto G., Prima lezione di sociolinguistica, Editori laterza, Roma-Bari, 2004.

Camilleri A.,\& De Mauro T., La lingua batte dove il dente duole, Editori laterza, Roma-Bari, novembre 2013.

Crespi F., Le vie della sociologia, Bologna: il Mulino, 1985.

Hudson R., Sociolinguistika, Dituria, 2002.

Klein G., Nozioni e strumenti di sociolinguistica. Con esercitazioni e glossario, Aracne, Roma, dicembre 2006.

Orletti F., La conversazione diseguale. Potere e interazione. Roma, Carocci, 2000.

Shkurtaj Gj., Sociolinguistika, shblu, Tiranë, 2003. 